\title{
Research on the Principle of Minimal Incompatibility for Fuzzy Reasoning
}

\author{
Lihua $\mathrm{Fu}^{1}$ Huacan $\mathrm{He}^{2}$ \\ ${ }^{1}$ College of Computer Science, Beijing University of Technology, Beijing 100022, P. R. China \\ ${ }^{2}$ College of Computer Science, Northwestern Polytechnical University, Xi'an 710072, P. R. China
}

\begin{abstract}
Based on the concept of incompatible factor, this paper introduces further the principle of minimal incompatibility, and use this principle to improve the triple I method. In addition, by the principles of maximal support and minimal incompatibility the solutions of the problems of Fuzzy Modus Ponens and Fuzzy Modus Tollens are unified. The reason why the new method is more reasonable than the triple I method is analyzed, and the new method is generalized by considering two different types of implication operators.
\end{abstract}

Keywords: Fuzzy reasoning, CRI method, Triple I method, Incompatible factor, The least incompatibility

\section{Introduction}

As is well known, fuzzy reasoning now has become a theoretical basis and an important method for the design and analysis of fuzzy controller, and it has found a considerable number of successful industrial applications in some fields such as intelligent control [1]. In 1973, the Fuzzy Modus Ponens(FMP) was introduced for the first time by Zadeh [3], and developed by Mamdani [4], so that it becomes the famous CRI method (Compositional Rule of Inference) [5]. As the fundamental method of fuzzy reasoning, the CRI method has been widely adopted and many researchers have generalized it in many different ways[6]-[8].

After making many detailed researches on the method of CRI, Wang pointed out some shortcomings about it and proposed a kind of new method for fuzzy reasoning, called as triple I method [9]-[10], which can be considered as a reasonable improvement to Zadeh's CRI method. However, by some theoretical analyses we discover that the triple I method also has some shortcomings. For example, it simply considers the minimal good set [10] as the result of FMP but has no reasonable explanation, and the result given by it may be not a reasonable one in some cases. In this paper, the principle of minimal incompatibility is proposed based on the concept of incompatible factor [11], and the result of fuzzy reasoning is renewedly given by it.

\section{Fundamental concepts}

\subsection{Fuzzy modus ponens(FMP)}

In the fundamental research on fuzzy reasoning, the basic model of FMP can be represented as follows [6]:

\begin{tabular}{lr}
$\begin{array}{l}\text { rule } \\
\text { for given }\end{array}$ & $\begin{array}{r}A \rightarrow B \\
A^{*}\end{array}$ \\
\hline to determine & $B^{*}$
\end{tabular}

where $A$ and $A^{*}$ are the fuzzy sets in domain $X$, and $B$ and $B^{*}$ are the fuzzy sets in domain $Y$.

\subsection{The CRI method and the triple I method}

\subsubsection{The CRI method}

To get the fuzzy set $B^{*}$ in Eq.(1), various methods have been carried out, but the Zadeh's CRI method is the most typical.

According to the CRI method, the fuzzy set $B^{*}$ in Eq.(1) may be determined by

$$
\begin{aligned}
B^{*}(y) & =A^{*}(x) \circ R_{Z}(A(x), B(y)) \\
& =\sup _{x \in X}\left[A *(x) \wedge R_{Z}(A(x), B(y))\right]
\end{aligned}
$$

where the variable $R_{\mathrm{Z}}:[0,1]^{2} \rightarrow[0,1]$ is Zadeh's implication operator defined as

$$
R_{z}(a, b)=a^{\prime} \vee(a \wedge b)
$$

where $a^{\prime}=1-a, a \in[0,1]$. Notice that there are two kinds of fuzzy logic operators in Eq.(2), the compound operator and the implication operator " $R_{Z}$ ". These operators have been defined by various different methods [12].

CRI has been widely applied successfully in various fields of industrial control. However, from the standpoint of logic semantics, there exist several 
problems in applying the method of CRI, which are represented as follows [10]:

1) The approach does not possess the property of reductivity.

2) CRI is such an implication method that adopts fuzzy reasoning only once, and other implications are simply replaced by the compound method.

\subsubsection{The triple I method}

To improve the method of CRI, the triple I method with total inference rules of fuzzy reasoning was proposed by Wang[10], of which the basic idea can be summarized as follows.

For $A, A^{*} \in F(X)$ and $B \in F(Y)$, the fuzzy set $B^{*}$ in Eq.(1) is the minimal fuzzy set of $F(Y)$ so that $A \rightarrow B$ completely supports $A^{*} \rightarrow B^{*}$, that is,

$$
M(x, y)=(A(x) \rightarrow B(y)) \rightarrow\left(A^{*}(x) \rightarrow B^{*}(y)\right)
$$

has the maximal possible value whenever $x \in X$ and $y \in Y$, where $F(X)$ and $F(Y)$ denote, respectively, the collections of all fuzzy subsets of $X$ and $Y$.

For the sake of convenience, the definition of good set is given as follows.

Definition 1. (Good Set)[10] For $A, A^{*} \in F(X)$ and $B \in F(Y)$, if the fuzzy set $B^{*}$ makes $M(x, y)$ in Eq.(5) take its maximal possible value, then $B^{*}$ is called good set, where $B^{*} \in F(Y)$.

\subsection{Main properties of implication operators}

Most of the definitions of the implication operator have been based on satisfaction to a number of properties associated with the classical binary implication operator, such as the famous D-P conditions [8]:

I1: if $a \leq a^{\prime}$ then $I(a, b) \geq I\left(a^{\prime}, b\right)$;

I2: if $b \geq b^{\prime}$ then $I(a, b) \geq I\left(a, b^{\prime}\right)$;

I3: $I(0, b)=1$;

I4: $I(1, b)=b$;

I5: $I(a, b) \geq b$;

I6: $I(a, a)=1$;

I7: $I(a, I(b, c))=I(b, I(a, \mathbf{c}))$;

I8: $I(a, b)=1$ if and only if $a \leq b$;

I9: $I(a, b)=I(n(b), n(a))$ for some strong negation

$n$;

I10: $I$ is continuous.

Due to their different definitions, they satisfy some different properties of D-P conditions. For example, $R_{K D}$ and $R_{R}$ satisfy the properties I1-I5, I7 and I9-I10, $R_{L u}$ satisfies I1-I10, and $R_{Z}$ satisfies the ones I2-I4 and I10.

\section{Incompatible factor}

All inferences of the triple I method are represented as implication operators, and the method has fully considered the completely supporting relation between " $A^{*}(x) \rightarrow B^{*}(y)$ " and " $A(x) \rightarrow B(y)$ ". Apparently, the idea of reasoning has greatly improved the CRI method, which adopts fuzzy reasoning only once, and other implications are simply replaced by the compound method.

However, it seems some farfetched that the minimal good set is considered as the fuzzy set $B^{*}$ in Eq.(1) in the triple I method. Furthermore, whether it is the result of FMP needs further investigation. There may be many good sets, for example, the maximal fuzzy set $1_{y}$ of $F(Y)$, which takes the constant 1 when $R$ satisfies I2. However, it is quite evident that $\operatorname{such} B^{*}$ is not reasonable because it does not propose any helpful information[14]. So the triple I method considers the minimal one as $B^{*}$ in Eq.(1)[14], but does not give a reasonable explanation.

When $B^{*} \in F(Y)$ is a good set and satisfies the following inequation: $R\left(A^{*}(x), B^{*}(y)\right)>R(A(x), B(y))$, that is, $A^{*}$ supports $B^{*}$ is more than $A$ supports $B$, this process of reasoning is considered hazardous. Contrarily, it will be considered conservative if $B^{*}$ satisfies the inequation: $R\left(A^{*}(x), B^{*}(y)\right)<R(A(x)$, $B(y))$.

Based on the above analysis, we can come to the conclusion that the fuzzy set $B^{*}$ in Eq.(1) not only should be a good set, that is, it should make $A \rightarrow B$ completely support $A^{*} \rightarrow B^{*}$, but make the extent to which $A^{*}$ supports $B^{*}$ is as close to the one to which $A$ supports $B$ as possible. Hence, we proposed the concept of incompatible factor as follows.

Definition 2. (Incompatible Factor)[11] Suppose that $X$ and $Y$ are nonempty sets, $A, A^{*} \in F(X), B, B^{*} \in F(Y)$. The fuzzy set $B^{*}$ in Eq.(1) has the degree of incompatibility $|r|$,

$$
r=R\left(A^{*}(x), B^{*}(y)\right)-R(A(x), B(y)) .
$$

Remark: If the incompatible factor $r$ is positive, then the reasoning is hazardous. Contrarily, it is conservative. Accordingly, the degree of incompatibility $|r|$ expresses the degree of hazard or conservation. Apparently, the fuzzy set $B^{*}$ in Eq.(1) should be make the degree of incompatibility $|r|$ as small as possible.

Definition 3. (Principle of Triple $I^{*} F M P$ ) Suppose that $X$ and $Y$ are nonempty sets, $A, A^{*} \in F(X), B$, $B^{*} \in F(Y)$. The fuzzy set $B^{*}$ in Eq.(1) is the one of $\mathrm{F}(\mathrm{Y})$ satisfying the following two principles:

1) (The principle of maximal support) $B^{*}$ is the fuzzy set of $F(Y)$ which makes $A \rightarrow B$ completely support $A^{*} \rightarrow B^{*}$, that is, Eq.(5) has the maximal possible value whenever $x \in X$ and $y \in Y$.

2) (The principle of minimal incompatibility) $B^{*}$ is the fuzzy set of $F(Y)$ which has the minimal 
incompatibility, that is, $|r|$ takes the minimal possible value.

According to the above-mentioned principle, we will discuss in the two cases by considering two different types of implication operators.

\section{Analysis on some examples}

\subsection{Implication operators satisfying the property 18}

For the implication operators satisfying the property I8, $B^{*}$ satisfying the principle of maximal support makes $\mathrm{M}(\mathrm{x}, \mathrm{y})$ in Eq.(5) take its maximum 1 and the inequation: $R\left(A^{*}(x), B^{*}(y)\right) \geq R(A(x), B(y))$ hold. It can be seen that some of these $B^{*}$ make $A^{*}$ support $B^{*}$ more than $A$ support $B$, so this process of reasoning is considered hazardous.

In fact, these implication operators satisfy the property $\mathrm{I} 2$, that is, the bigger $B^{*}$ becomes, the bigger $R\left(A^{*}(x), B^{*}(y)\right)$ does. In order to control the hazardous trend, we should decrease the value of $B^{*}$, so that the value of $\left(A^{*}(x) \rightarrow B^{*}(y)\right)$ goes to the one of $(A(x) \rightarrow$ $B(y))$. Apparently, such $B^{*}$ makes the extent to which $A^{*}$ supports $B^{*}$ go to the one to which $A$ supports $B$, so it is a reasonable result of FMP. Furthermore, its according degree of incompatibility $|r|$ goes to 0 , too, that is, it satisfies the principle of minimal incompatibility.

According to the above analysis, it can be seen that as adopting the implication operators satisfying the property I8, the result of the principle of triple $I^{*}$ FMP is the minimal good set, which is consistent with the one of the triple I FMP, but it has more reasonable explanation.

Example 1. Suppose that the variable $R:[0,1]^{2} \rightarrow[0,1]$ is Łukasiewicz's implication operator defined as:

$$
R_{L u}(a, b)=\left(a^{\prime}+b\right) \wedge 1
$$

For a fixed $y \in Y$, let

$$
E_{y}=\left\{x \in X \mid\left(A^{*}(x)\right)^{\prime}<R_{L u}(A(x), B(y))\right\} .
$$

We shall discuss in two possible cases as follows.

1) If $x \notin E_{y}$, then

$$
\left(A^{*}(x)\right)^{\prime} \geq R_{L u}(A(x), B(y)) .
$$

By the definition in Eq.(6), we know $R_{L u}\left(A^{*}(x), B^{*}(y)\right) \geq\left(A^{*}(x)\right)^{\prime}$, and using Eq.(7), we have

$$
R_{L u}\left(A^{*}(x), B^{*}(y)\right) \geq R_{L u}(A(x), B(y)) .
$$

Thus, any $B^{*} \in F(Y)$ make $M(x, y)$ in Eq.(5) take its maximum 1and $r=R_{L u}\left(A^{*}(x), B^{*}(y)\right)-R_{L u}(A(x)$, $B(y)) \geq 0$. To make $r$ take its minimum, $B^{*}(y)=0$. 2) If $x \in E_{y}$, then

$$
\left(A^{*}(x)\right)^{\prime}<R_{L u}(A(x), B(y)) .
$$

By the definition in Eq.(6), in order to make $M(x$, $y$ ) in Eq.(5) take its maximum 1, we have

$$
\begin{aligned}
R_{L u}\left(A^{*}(x), B^{*}(y)\right) & =\left(A^{* \prime}(x)+B^{*}(y)\right) \wedge 1 \\
& \geq R_{L u}(A(x), B(y))
\end{aligned}
$$

Furthermore, to make $r$ take its minimum, which is positive, we have

$$
B^{*}(y)=\sup _{x \in E_{y}}\left\{R_{L u}(A(x), B(y))-\left(A^{*}(x)\right)^{\prime}\right\} .
$$

According to the above analyses of 1) and 2), we have

$$
B^{*}(y)=\sup _{x \in E_{y}}\left\{R_{L u}(A(x), B(y))-\left(A^{*}(x)\right)^{\prime}\right\} .
$$

It is noteworthy that the expression Eq.(9) of the FMP conclusion $B^{*}$ is consistent with the one of the triple I FMP, but it has more reasonable explanation.

\subsection{Implication operators not satisfying the property 18}

For the implication operators not satisfying the property $\mathrm{I} 8$, we can still use the principle of triple $I^{*}$ $F M P$ to seek an optimal $B^{*} \in F(Y)$ which makes $M(x, y)$ take its maximum, but this maximum usually is not the constant 1. Furthermore, for these implication operators do not satisfy the property $\mathrm{I} 8$, such $B^{*} \in F(Y)$ which make $M(x, y)$ take its maximum do not make $R\left(A^{*}(x), B^{*}(y)\right) \geq R(A(x), B(y))$ always hold. Hence, for this kind of implication operators it is not quite reasonable that the minimal good set is still considered as the FMP conclusion $B^{*}$ by the principle of triple I FMP.

In order to illustrate the question more clearly, we will use Zadeh's implication operator $R_{Z}$ as an example to make a detailed discuss about it.

Now, we will discuss when $R_{Z}$ can take its maximum.

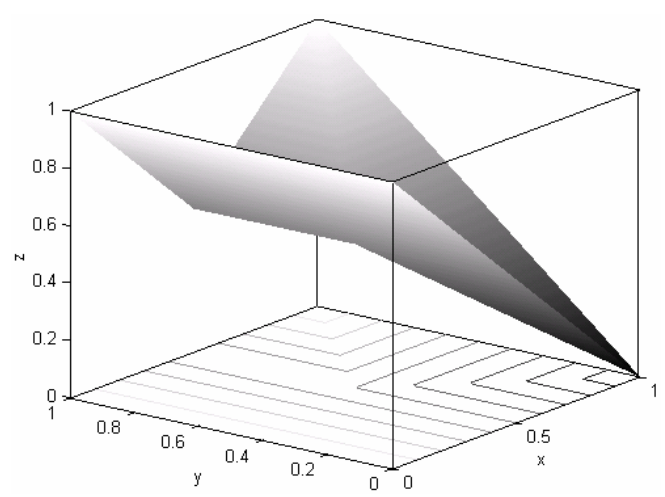

Fig. 1: The Figure of $R_{z}$ 
So we can draw the conclusion that if $a \leq b, R_{\mathrm{Z}}(a$, $b)$ always takes its maximum, and if $a>b, R_{\mathrm{Z}}(a, b)$ takes this one only when $a \leq 0.5$.

Example 2. Suppose that the variable $R:[0,1]^{2} \rightarrow[0$, 1] is Zadeh's implication operator $R_{Z}$ defined in Eq.(4). For a fixed $y \in Y$, let

$$
E_{y}=\left\{x \in X \mid R_{Z}(A(x), B(y)) \leq 0.5\right\} .
$$

$F_{y}=\left\{x \in E_{y} \mid\left(A^{*}(x)\right)^{\prime}<R_{Z}(A(x), B(y))\right\}$.

$G_{y}=\left\{x \in E_{y} \mid\left(A^{*}(x)\right)^{\prime} \geq R_{Z}(A(x), B(y))\right\}$.

$H_{y}=\left\{x \notin E_{y} \mid\left(A^{*}(x)\right)^{\prime}<R_{Z}(A(x), B(y))\right\}$.

$I_{y}=\left\{x \in H_{y} \mid A^{*}(x) \geq R_{Z}(A(x), B(y))\right\}$.

$K_{y}=\left\{x \in H_{y} \mid A^{*}(x)<R_{Z}(A(x), B(y))\right\}$.

$L_{y}=\left\{x \in K_{y} \mid A *(x) \leq 0.5\right\}$.

$M_{y}=\left\{x \in K_{y} \mid A^{*}(x)>0.5\right\}$.

$N_{y}=\left\{x \notin E_{y} \mid\left(A^{*}(x)\right)^{\prime} \geq R_{z}(A(x), B(y))\right\}$.

According to Zadeh's implication operator $R_{Z}$, we have

$$
\begin{array}{r}
M(x, y)=R_{Z}\left(R_{Z}(A(x), B(y)), R_{Z}\left(A^{*}(x), B^{*}(y)\right)\right) \\
\quad=R_{Z}^{\prime}(A(x), B(y)) \vee\left[R_{Z}(A(x), B(y)) \wedge R_{Z}\left(A^{*}(x), B^{*}(y)\right)\right]
\end{array}
$$

1) If $x \in E_{y}$, that is, $R_{Z}(A(x), B(y)) \leq 0.5$, then Eq.(10) can take its maximum in the following two cases:

$$
\begin{gathered}
R_{Z}\left(A^{*}(x), B^{*}(y)\right) \geq R_{Z}(A(x), B(y)) \\
\text { or }
\end{gathered}
$$$$
R_{\mathrm{Z}}\left(A^{*}(x), B^{*}(y)\right)<R_{\mathrm{Z}}(A(x), B(y))
$$

A) If $R_{Z}\left(A^{*}(x), B^{*}(y)\right) \geq R_{Z}(A(x), B(y))$, then

$$
\left(A^{*}(x)\right)^{\prime} \vee\left(A^{*}(x) \wedge B^{*}(y)\right) \geq R_{Z}(A(x), B(y))
$$

a) For $x \in F_{y}$, in order to make Eq.(11) hold, we have $B^{*}(y) \geq R_{Z}(A(x), B(y))$. Furthermore, to make the incompatible factor $r$ take its minimum, which is positive, we have

$$
B^{*}(y)=\sup _{x \in F_{y}}\left\{R_{Z}(A(x), B(y))\right\}
$$

b) If $x \in G_{y}$, that is, $\left(A^{*}(x)\right)^{\prime} \geq R_{Z}(A(x), B(y))$, then Eq.(11) always holds. Furthermore, to make the incompatible factor $r$ take its minimum, which is positive, we have

$$
B^{*}(y)=0
$$

B) If $R_{Z}\left(A^{*}(x), B^{*}(y)\right)<R_{Z}(A(x), B(y))$, then $\left(A^{*}(x)\right)^{\prime} \vee\left(A^{*}(x) \wedge B^{*}(y)\right)<R_{Z}(A(x), B(y))$

a) For $x \in F_{y}$, in order to make Eq.(14), we have $B^{*}(y)<R_{Z}(A(x), B(y))$. Furthermore, to make the incompatible factor $r$ go to 0 , which is negative, we have

$$
B *(y)=\inf _{x \in F_{y}}\left\{R_{Z}(A(x), B(y))\right\}
$$

b) If $x \in G_{y}$, that is, $\left(A^{*}(x)\right)^{\prime} \geq R_{Z}(A(x), B(y))$, then Eq.(14) impossibly holds.

2) If $x \notin^{\notin}$, that is, $R_{Z}(A(x), B(y))>0.5$, then Eq.(10) can take its maximum only in the case: $R_{Z}\left(A^{*}(x)\right.$, $\left.B^{*}(y)\right) \geq R_{Z}(A(x), B(y))$, that is,

$$
\left(A^{*}(x)\right)^{\prime} \vee\left(A^{*}(x) \wedge B^{*}(y)\right) \geq R_{Z}(A(x), B(y))
$$

A) If $x \in I_{y}$, then $\left(A^{*}(x)\right)^{\prime}<R_{Z}(A(x), B(y))$ and $A^{*}(x)$ $\geq \quad R_{Z}(A(x), B(y))$ hold. In order to make Eq.(16) hold, we have $B^{*}(y) \geq R_{Z}(A(x), B(y))$. Furthermore, to make the incompatible factor $r$ take its minimum, which is positive, we have

$$
B *(y)=\sup _{x \in I_{y}}\left\{R_{Z}(A(x), B(y))\right\}
$$

B) If $x \in K_{y}$, that is, $\left(A^{*}(x)\right)^{\prime}<R_{\mathrm{Z}}(A(x), B(y))$ and $A^{*}(x)<R_{Z}(A(x), \quad B(y))$ hold, then Eq.(16) impossibly holds. Hence, in this case $R_{Z}\left(A^{*}(x)\right.$, $\left.B^{*}(y)\right)<R_{Z}(A(x), B(y))$ must hold, then Eq.(10) can take its maximum only when $R_{\mathrm{Z}}\left(A^{*}(x), B^{*}(y)\right)$ takes its maximum. Similarly, we will discuss when $R_{Z}\left(A^{*}(x), B^{*}(y)\right)$ can take its maximum.

a) If $x \in L_{y}$, that is, $A^{*}(x) \leq 0.5$, we have $A^{*}(x) \leq$ $0.5 \leq\left(A^{*}(x)\right)^{\prime}<R_{Z}(A(x), B(y))$. In this case, $R_{\mathrm{Z}}\left(A^{*}(x), B^{*}(y)\right)$ always takes its maximum $\left(A^{*}(x)\right)^{\prime}$

b) If $x \in M_{y}$, that is, $A^{*}(x)>0.5$, we have $\left(A^{*}(x)\right)^{\prime}$ $<0.5<A^{*}(x)<R_{\mathrm{Z}}(A(x), B(y))$. When $B^{*}(y) \geq$ $A^{*}(x), R_{Z}\left(A^{*}(x), B^{*}(y)\right)$ can take its maximum $A^{*}(x)$, so we have

$$
B^{*}(y)=\sup _{x \in M_{y}}\left\{A^{*}(x)\right\}
$$

C) If $x \in N_{y}$, that is, $\left(A^{*}(x)\right)^{\prime} \geq R_{Z}(A(x), B(y))$, then Eq.(16) always holds. Furthermore, to make the incompatible factor $r$ take its minimum, which is positive, we have

$$
B^{*}(y)=0
$$

\section{Fuzzy modus tollens (FMT)}

The opposite form of fuzzy modus ponens(FMP) is fuzzy modus tollens(FMT), which can be expressed as

$$
\begin{array}{lr}
\text { rule } & A \rightarrow B \\
\text { for given } & B^{*} \\
\hline
\end{array}
$$

to determine $A^{*}$

where $A$ and $A^{*}$ are fuzzy sets in domain $X$, and $B$ and $B^{*}$ are the fuzzy sets in the domain $Y$.

If $R$ satisfies the property I1, then Wang has proposed the principle of triple I FMT as follows [10]:

Definition 4. (The principle of triple I FMT) [10] Suppose that $X$ and $Y$ are nonempty sets, $A \in F(X), B$, $B^{*} \in F(Y)$. Then $A^{*} \in F(X)$ satisfying Eq.(20) is the maximal fuzzy set that makes $M(x, y)$ in Eq.(5) take its maximal possible value whenever $x \in X$ and $y \in Y$.

Similarly, for the problem of FMT, the following principle can be obtained using the principle of minimal incompatibility.

Definition 5. (The principle of triple $I^{*} F M T$ ) Suppose that $X$ and $Y$ are nonempty sets, $A, A^{*} \in F(X), B$, 
$B^{*} \in F(Y)$. The fuzzy set $A^{*}$ in Eq.(20) is the one of $F(X)$ satisfying the following two principles:

1) (The principle of maximal support) $A^{*}$ is the fuzzy set of $F(X)$ which makes $A \rightarrow B$ completely support $A^{*} \rightarrow B^{*}$, that is, Eq.(5) has the maximal possible value whenever $x \in X$ and $y \in Y$.

2) (The principle of minimal incompatibility) $A^{*}$ is the fuzzy set of $F(X)$ which has the minimal incompatibility, that is, $|r|$ takes its minimal possible value.

By making a comparison between the Def. 4 and the Def.5, it can be seen that the principle of minimal incompatibility can not only give a more reasonable result of FMP or FMT but also unify the solvings of them, that is, we need not remember that the result of FMP is the minimal good set, but the one of FMT is the maximal one.

\section{Conclusions}

In this paper, based on the concept of incompatible factor, the principle of minimal incompatibility is introduced. The triple I method is improved which simply considers the minimal good set as the result of FMP and the more reasonable form is also given. In addition, by the principles of maximal support and minimal incompatibility the solvings of the problems of FMP and FMT are unified, so that it is more convenient to solve them.

What are the sufficient conditions for that these methods are P-reductors? How does the principle of minimal incompatibility work in the reversed triple I method [15]-[16] of fuzzy reasoning? These problems will be further studied elsewhere.

\section{References}

[1] J. Yen and R. Langari, Fuzzy Logic: Intelligence, Control, and Information. Upper Saddle River, NJ: Prentice-Hall, 1999.

[2] Mingsheng Ying, Implication operators in fuzzy logic. IEEE Trans. on Fuzzy Systems, 10(1): 8891, 2002.

[3] L. A. Zadeh, Outline of a new approach to the analysis of complex systems and decision processes. IEEE Trans SMC, 1: 28-44, 1973.

[4] E H. Mamdani, Application of fuzzy logic to approximate reasoning using linguistic systems. IEEE Trans. on Computers, 26: 1182-1191, 1977.

[5] L. A. Zadeh, The concept of linguistic variable and its application to approximate reasoning. part 1-3[J]. Information Sciences, 8: 199-249; 301-357; 9: 43-80, 1975.
[6] B.K. Zhou, G.J. Wang, Triple-I algorithm under different implication operators. Journal of Shaanxi Normal University(Natural Science Edition), 26(4): 1-5, 1998 (in Chinese).

[7] Mizumoto M, Zimmermann H J. Comparison of fuzzy reasoning methods. Fuzzy Sets and Systems, 8: 253-283, 1982.

[8] Dubois D, Prade H. Fuzzy sets in approximate reasoning. Part 1:Inference with possibility distributions. Fuzzy Sets and Systems, 41(1): 153203, 1991.

[9] G.J. Wang, On the logic foundation of fuzzy reasoning. Information Sciences, 117: 47-88, 1999.

[10] G.J. Wang, Full implication triple I method for fuzzy reasoning. Science in China (Series E), 29(1): 43-53, 1999.

[11] L.H. Fu, H.C. He, Research on the incompatible factor of fuzzy reasoning, Computer Sciences, 31(2): 98-100, 2004 (in Chinese).

[12] L.X. Wang, A course in fuzzy systems and control, Prentice Hall PTR, Upper Saddle River, 1997.

[13] W.M. Wu, Principles and methods of fuzzy reasoning, Guizhou Science and Technology Press, Guiyang, 1994 (in Chinese).

[14] G.J. Wang, Non-classical mathematical logic and approximate reasoning, Science Press, Beijing, 2000 (in Chinese).

[15] S.J. Song, C. Wu, Reversed triple I method of fuzzy reasoning, Science in China (Series E), 32(2): 230-246, 2002.

[16] S.J. Song, C.B. Feng, E.S. Lee, Triple I method of fuzzy reasoning, Computers and Mathematics with Applications, 44: 1567-1579, 2002. 